JETP Letters, 2010, Vol. 92, No. 3, pp. 125-129.

\title{
On the collisions between particles in the vicinity of rotating black holes ${ }^{1)}$
}

\author{
A. A. Grib ${ }^{a}$, Yu. V.Pavlov \\ ${ }^{a}$ Theoretical Physics and Astronomy Department, The Herzen University, 48, Moika, St. Petersburg, 191186, Russia \\ e-mail: andrei_grib@mail.ru \\ ${ }^{b}$ Institute of Problems in Mechanical Engineering, RAS, 61 Bolshoy, V.O., St. Petersburg, 199178, Russia \\ e-mail: yuri.pavlov@mail.ru
}

Submitted 9 June 2010

\begin{abstract}
Scattering of particles in the gravitational field of rotating black holes is considered. It is shown that scattering energy of particles in the centre of mass system can obtain very large values not only for extremal black holes but also for nonextremal ones. Extraction of energy after the collision is investigated. It is shown that due to the Penrose process the energy of the particle escaping the hole at infinity can be large. Contradictions in the problem of getting high energetic particles escaping the black hole are resolved.
\end{abstract}

In [1] we put the hypothesis that active galactic nuclei can be the sources of ultrahigh energy particles in cosmic rays observed recently by the AUGER group (see [2]) due to the processes of converting dark matter formed by superheavy neutral particles into visible particles - quarks, leptons (neutrinos), photons. If active galactic nuclei are rotating black holes then in [1] we discussed the idea that "This black hole acts as a cosmic supercollider in which superheavy particles of dark 'matter are accelerated close to the horizon to the Grand Unification energies and can be scattering in collisions." 'It was also shown 3 that in Penrose process 4 dark matter particle can decay on two particles, one with the negative energy, the other with the positive one and particles of very high energy of the Grand Unification order can escape the black hole. Then these particles due to interaction with photons close to the black hole will loose energy analogously up to the Greisen-Zatsepin'Kuzmin limit in cosmology [5, 6.

First calculations of the scattering of particles in the ergosphere of the rotating black hole, taking into account the Penrose process, with the result that particles with high energy can escape the black hole, were made in 7, 8. Recently in 9 it was shown that for the rotating black hole (if it is the critical one) the energy of scattering is unlimited. The result of [9] was criticized in [10, 11] in the sense that it does not occur in nature. The authors of [10, 11] claimed that if the black hole is not a critical rotating black hole so that its dimensionless angular momentum $A \neq 1$ but $A=0.998$ then the energy is limited.

In this paper we show that the energy of scattering in the centre of mass system can be still unlimited in

1) On particles collisions in the vicinity of rotating black holes, Pis'ma v ZhETF, vol. 92, iss. 3, (2010) 147-151. the cases of multiple scattering. In the first part we calculate this energy, reproduce the results of 9, 10, 11] and show that in some cases (multiple scattering) the results of [10, 11] on the limitations of the scattering energy for nonextremal black holes are not valid.

In the second part we obtain the results for the extraction of the energy after collision in the field of the Kerr's metric. It occurs that the Penrose process plays important role for getting larger energies of particles at infinity. Our calculations show that the conclusion of 11 about the impossibility of getting at infinity the energy larger than the initial one in particle collisions close to the black hole is wrong.

The system of units $G=c=1$ is used in the paper.

\section{THE ENERGY OF COLLISION IN THE FIELD OF BLACK HOLES}

Let us consider particles falling on the rotating chargeless black hole. The Kerr's metric of the rotating black hole in Boyer-Lindquist coordinates has the form

$$
\begin{gathered}
d s^{2}=d t^{2}-\frac{2 M r\left(d t-a \sin ^{2} \theta d \varphi\right)^{2}}{r^{2}+a^{2} \cos ^{2} \theta} \\
-\left(r^{2}+a^{2} \cos ^{2} \theta\right)\left(\frac{d r^{2}}{\Delta}+d \theta^{2}\right)-\left(r^{2}+a^{2}\right) \sin ^{2} \theta d \varphi^{2},
\end{gathered}
$$

where

$$
\Delta=r^{2}-2 M r+a^{2},
$$

$M$ is the mass of the black hole, $J=a M$ is angular momentum. In the case $a=0$ the metric (1D) describes the static chargeless black hole in Schwarzschild coordinates. The event horizon for the Kerr's black hole corresponds to the value

$$
r=r_{H} \equiv M+\sqrt{M^{2}-a^{2}} .
$$


The Cauchy horizon is

$$
r=r_{C} \equiv M-\sqrt{M^{2}-a^{2}} .
$$

The surface called "the static limit" is defined by the expression

$$
r=r_{0} \equiv M+\sqrt{M^{2}-a^{2} \cos ^{2} \theta} .
$$

The region of space-time between the horizon and the static limit is ergosphere.

For equatorial $(\theta=\pi / 2)$ geodesics in Kerr's metric (11) one obtains $([12, \S 61)$ :

$$
\begin{gathered}
\frac{d t}{d \tau}=\frac{1}{\Delta}\left[\left(r^{2}+a^{2}+\frac{2 M a^{2}}{r}\right) \varepsilon-\frac{2 M a}{r} L\right], \\
\frac{d \varphi}{d \tau}=\frac{1}{\Delta}\left[\frac{2 M a}{r} \varepsilon+\left(1-\frac{2 M}{r}\right) L\right], \\
\left(\frac{d r}{d \tau}\right)^{2}=\varepsilon^{2}+\frac{2 M}{r^{3}}(a \varepsilon-L)^{2}+\frac{a^{2} \varepsilon^{2}-L^{2}}{r^{2}}-\frac{\Delta}{r^{2}} \delta_{1},
\end{gathered}
$$

where $\delta_{1}=1$ for timelike geodesics $\left(\delta_{1}=0\right.$ for isotropic geodesics), $\tau$ is the proper time of the moving particle, $\varepsilon=$ const is the specific energy: the particle with rest mass $m$ has the energy $\varepsilon m$ in the gravitational field (1); $L m=$ const is the angular momentum of the particle relative to the axis orthogonal to the plane of movement.

We denote $x=r / M, x_{H}=r_{H} / M, x_{C}=r_{C} / M$, $A=a / M, \quad l_{n}=L_{n} / M, \quad \Delta_{x}=x^{2}-2 x+A^{2}$. For the energy in the centre of mass frame of two colliding particles with angular momenta $L_{1}, L_{2}$, which are nonrelativistic at infinity $\left(\varepsilon_{1}=\varepsilon_{2}=1\right)$ and are moving in Kerr's metric using (11), (6) $-(8)$ one obtains 9 :

$$
\begin{gathered}
\frac{E_{\mathrm{c} . \mathrm{m} .}^{2}}{2 m^{2}}=\frac{1}{x \Delta_{x}}\left[2 x^{2}(x-1)+l_{1} l_{2}(2-x)\right. \\
+2 A^{2}(x+1)-2 A\left(l_{1}+l_{2}\right) \\
\left.-\sqrt{\left(2 x^{2}+2\left(l_{1}-A\right)^{2}-l_{1}^{2} x\right)\left(2 x^{2}+2\left(l_{2}-A\right)^{2}-l_{2}^{2} x\right)}\right] .
\end{gathered}
$$

To find the limit $r \rightarrow r_{H}$ for the black hole with a given angular momentum $A$ one must take in (9) $x=x_{H}+\alpha$ with $\alpha \rightarrow 0$ and do calculations up to the order $\alpha^{2}$. Taking into account $A^{2}=x_{H} x_{C}, x_{H}+x_{C}=2$, after resolution of uncertainties in the limit $\alpha \rightarrow 0$ one obtains

$$
\frac{E_{\mathrm{c} . \mathrm{m} .}\left(r \rightarrow r_{H}\right)}{2 m}=\sqrt{1+\frac{\left(l_{1}-l_{2}\right)^{2}}{2 x_{C}\left(l_{1}-l_{H}\right)\left(l_{2}-l_{H}\right)}},
$$

where $l_{H}=2 x_{H} / A$.
For the extremal black hole $A=x_{H}=1, l_{H}=2$ and the expression (10) is divergent when the dimensionless angular momentum of one of the falling into the black hole particles $l \rightarrow 2$. The scattering energy in the centre of mass system is increasing without limit [9].

Let's note, that to get the collision with infinite energy one needs the infinite interval of as coordinate as proper time of the free falling particle. Really, from Eqs. (6), (8) for a particle with dimensionless angular momentum $l$ and specific energy $\varepsilon=1$ falling from some point $r_{0}=x_{0} M$ to the point $r_{f}=x_{f} M>r_{H}$ one obtains for the coordinate time (proper time of the observer at rest far from the black hole)

$$
\Delta t=M \int_{x_{f}}^{x_{0}} \frac{\sqrt{x}\left(x^{3}+A^{2} x+2 A(A-l)\right) d x}{\left(x-x_{H}\right)\left(x-x_{C}\right) \sqrt{2 x^{2}-l^{2} x+2(A-l)^{2}}} .
$$

For the interval of proper time of the free falling to the black hole particle one obtains from (8)

$$
\Delta \tau=M \int_{x_{f}}^{x_{0}} \frac{x^{3 / 2} d x}{\sqrt{2 x^{2}-l^{2} x+2(A-l)^{2}}} .
$$

In extremal case $(A=1, l=2)$ the integrals (11), (12) diverges for $x_{f} \rightarrow x_{H}=1$ and $\Delta t \approx M 2 \sqrt{2}\left(x_{f}-1\right)^{-1}$, $\Delta \tau \approx M\left|\ln \left(x_{f}-1\right)\right| / \sqrt{2}$ for $x_{f} \rightarrow 1$.

From (7), (8) for the angle of the particle falling in equatorial plane of the black hole one obtains

$$
\Delta \varphi=\int_{x_{f}}^{x_{0}} \frac{\sqrt{x}(x l+2(A-l)) d x}{\left(x-x_{H}\right)\left(x-x_{C}\right) \sqrt{2 x^{2}-l^{2} x+2(A-l)^{2}}} .
$$

If $A \neq 0$, then integral (13) is divergent for $x_{f} \rightarrow x_{H}$. In extremal case $(A=1, l=2) \Delta \varphi \approx \sqrt{2}\left(x_{f}-1\right)^{-1}$ for $x_{f} \rightarrow 1$. So before collision with infinitely large energy the particle must commit infinitely large number of rotations around the black hole.

Can one get the unlimited high energy of this scattering energy for the case of nonextremal black hole? Formula (8) leads to limitations on the possible values of the angular momentum of falling particles: the massive particle free falling in the black hole with dimensionless angular momentum $A$ being nonrelativistic at infinity $(\varepsilon=1)$ to achieve the horizon of the black hole must have angular momentum from the interval

$$
-2(1+\sqrt{1+A})=l_{L} \leq l \leq l_{R}=2(1+\sqrt{1-A}) .
$$

Putting the limiting values of angular momenta $l_{L}, l_{R}$ into the formula (10) one obtains the maximal 
values of the collision energy of particles freely falling from infinity

$$
\begin{gathered}
E_{\mathrm{c} . \mathrm{m} .}^{\mathrm{inf}}\left(r \rightarrow r_{H}\right)=\frac{2 m}{\sqrt[4]{1-A^{2}}} \\
\times \sqrt{\frac{1-A^{2}+(1+\sqrt{1+A}+\sqrt{1-A})^{2}}{1+\sqrt{1-A^{2}}}} .
\end{gathered}
$$

For $A=1-\epsilon$ with $\epsilon \rightarrow 0$ formula (15) gives:

$$
E_{\mathrm{c} . \mathrm{m} .}^{\inf }\left(r \rightarrow r_{H}\right) \sim 2\left(2^{1 / 4}+2^{-1 / 4}\right) \frac{m}{\epsilon^{1 / 4}} .
$$

So even for values close to the extremal $A=1$ of the rotating black hole $E_{\mathrm{c} . \mathrm{m} .}^{\mathrm{inf}} / m$ can be not very large as mentioned in [10, 11]. So for $A_{\max }=0.998$ considered as the maximal possible dimensionless angular momentum of the astrophysical black holes (see 13]), from (15) one obtains $E_{\text {c.m. }}^{\max } / m \approx 18.97$.

Does it mean that in real processes of particle scattering in the vicinity of the rotating nonextremal black holes the scattering energy is limited so that no Grand Unification or even Planckean energies can be obtained? Let us show that the answer is no! If one takes into account the possibility of multiple scattering so that the particle falling from infinity on the black hole with some fixed angular momentum changes its momentum in the result of interaction with particles in the accreting disc and after this is again scattering close to the horizon then the scattering energy can be unlimited.

The limiting value of the angular momentum of the particle close to the horizon of the black hole can be obtained from the condition of positive derivative in (6) $d t / d \tau>0$, i.e. going "forward" in time. So close to the horizon one has the condition $l<\varepsilon 2 x_{H} / A$ which for $\varepsilon=1$ gives the limiting value $l_{H}$.

From (8) one can obtain the permitted interval in $r$ for particles with $\varepsilon=1$ and angular momentum $l=l_{H}-\delta$. To do this one must put the left hand side of (8) to zero and find the roots:

$$
x_{1,2}=\frac{l^{2} \pm \sqrt{l^{4}-16(A-l)^{2}}}{4} .
$$

In the second order in $\delta$ close to the horizon one obtains

$$
l=l_{H}-\delta \Rightarrow x \lesssim x_{H}+\frac{\delta^{2} x_{C}^{2}}{4 x_{H} \sqrt{1-A^{2}}} .
$$

The effective potential defined by the right hand side of (8) leads to the following behaviour of the particle. If the particle goes from infinity to the black hole it can achieve the horizon if the inequality (14) is valid. However the scattering energy in the centre of mass frame given by (15) is not large. But if the particle is going not from the infinity but from some distance defined by (18) then due to the form of the potential it can have values of $l=l_{H}-\delta$ large than $l_{R}$ and fall on the horizon. If the particle falling from infinity with $l \leq l_{R}$ arrives to the region defined by (18) and here it interacts with other particles of the accretion disc or it decays into a lighter particle which gets an increased angular momentum $l_{1}=l_{H}-\delta$, then due to (10) the scattering energy in the centre of mass system is

$$
E_{\mathrm{c} . \mathrm{m} .} \approx \frac{m}{\sqrt{\delta}} \sqrt{\frac{2\left(l_{H}-l_{2}\right)}{1-\sqrt{1-A^{2}}}}
$$

and it increases without limit for $\delta \rightarrow 0$. For $A_{\max }=$ 0.998 and $l_{2}=l_{L}, \quad E_{\text {c.m. }} \approx 3.85 \mathrm{~m} / \sqrt{\delta}$.

Note that for rapidly rotating black holes $A=1-\epsilon$ the difference between $l_{H}$ and $l_{R}$ is not large

$$
\begin{aligned}
l_{H}-l_{R} & =2 \frac{\sqrt{1-A}}{A}(\sqrt{1-A}+\sqrt{1+A}-A) \\
& \approx 2(\sqrt{2}-1) \sqrt{\epsilon}, \quad \epsilon \rightarrow 0 .
\end{aligned}
$$

For $A_{\max }=0.998, \quad l_{H}-l_{R} \approx 0.04$ so the possibility of getting small additional angular momentum in interaction close to the horizon seems much probable. The probability of multiple scattering in the accretion disc depends on its particle density and is large for large density.

Here we consider the model when the gravitation of the accretion disc is treated as some perturbation much smaller than the gravitation of the black hole so it is not taken into account. One must also mention that "particles" are considered as elementary particles and not macroscopic bodies. So their "large" energy is limited by a Planckean value and we neglect back reaction of it on the Kerr's metric of the macroscopic black hole. Electromagnetic and gravitational radiation of the particle surely can change the picture but one needs exact calculations to see what will be the balance.

\section{THE EXTRACTION OF ENERGY AFTER THE COLLISION IN KERR'S METRIC}

Let us consider the case when there occurred for some $r>r_{H}$ interaction between particles with masses $m$, specific energies $\varepsilon_{1}, \varepsilon_{2}$, specific angular momenta $L_{1}, L_{2}$ falling into a black hole, so that two new particles with rest masses $\mu$ appeared, one of them (1) moved outside the black hole, the other (2) moved inside it. Denote the specific energies of new particles as $\varepsilon_{1 \mu}, \varepsilon_{2 \mu}$, their angular momenta (in units of $\mu$ ) as $L_{1 \mu}, L_{2 \mu}, v^{i}=d x^{i} / d s$ - their 4 -velocities. Consider 
particle movement in the equatorial plane of the rotating black hole.

Conservation laws in inelastic particle collisions for the energy and momentum lead to

$$
m\left(u_{(1)}^{i}+u_{(2)}^{i}\right)=\mu\left(v_{(1)}^{i}+v_{(2)}^{i}\right) .
$$

Equations (21) for $t$ and $\varphi$-components can be written as

$$
\begin{gathered}
m\left(\varepsilon_{1}+\varepsilon_{2}\right)=\mu\left(\varepsilon_{1 \mu}+\varepsilon_{2 \mu}\right), \\
m\left(L_{1}+L_{2}\right)=\mu\left(L_{1 \mu}+L_{2 \mu}\right),
\end{gathered}
$$

i.e. the sum of energies and angular momenta of colliding particles is conserved in the field of Kerr's black hole. The initial particles in our case were supposed to be nonrelativistic at infinity: $\varepsilon_{1}=\varepsilon_{2}=1$ and therefore for the $r$-component from (8) one obtains

$$
\begin{gathered}
-m\left[\sqrt{\frac{2 M}{r^{3}}\left(a-L_{1}\right)^{2}+\frac{2 M}{r}-\frac{L_{1}^{2}}{r^{2}}}\right. \\
\left.+\sqrt{\frac{2 M}{r^{3}}\left(a-L_{2}\right)^{2}+\frac{2 M}{r}-\frac{L_{2}^{2}}{r^{2}}}\right] \\
=\mu\left[\sqrt{\varepsilon_{1 \mu}^{2}+\frac{2 M}{r^{3}}\left(a \varepsilon_{1 \mu}-L_{1 \mu}\right)^{2}+\frac{a^{2} \varepsilon_{1 \mu}^{2}-L_{1 \mu}^{2}-\Delta}{r^{2}}}\right. \\
\left.-\sqrt{\varepsilon_{2 \mu}^{2}+\frac{2 M}{r^{3}}\left(a \varepsilon_{2 \mu}-L_{2 \mu}\right)^{2}+\frac{a^{2} \varepsilon_{2 \mu}^{2}-L_{2 \mu}^{2}-\Delta}{r^{2}}}\right] .
\end{gathered}
$$

The signs in (24) are put so that the initial particles and the particle (2) go inside the black hole while particle (1) goes outside the black hole. The values $\varepsilon_{1 \mu}, \varepsilon_{2 \mu}$ are constants on geodesics $(12, \S 61)$ so the problem of evaluation of the energy at infinity extracted from the black hole in collision reduces to a problem to find these values.

For the case when the collision takes place on the horizon of the black hole $\left(r \rightarrow r_{H}\right)$ the system (22)(24) can be solved exactly

$\varepsilon_{1 \mu}=\frac{A L_{1 \mu}}{2 r_{H}}, \varepsilon_{2 \mu}=\frac{2 m}{\mu}-\frac{A L_{1 \mu}}{2 r_{H}}, L_{2 \mu}=\frac{m}{\mu}\left(L_{1}+L_{2}\right)-L_{1 \mu}$.

In general case the system of three Eqs. (22)- (24) for four variables $\varepsilon_{1 \mu}, \varepsilon_{2 \mu}, L_{1 \mu}, L_{2 \mu}$ can be solved numerically for a fixed value of one variable (and fixed parameters $\left.m / \mu, L_{1} / M, L_{2} / M, a / M, r / M\right)$. The example of numerical solution is $\mu / m=0.3, l_{1}=$ $2.2, l_{2}=2.198, A=0.99, x=1.21, l_{1 \mu}=16.35, l_{2 \mu}=$ $-1.69, \varepsilon_{1 \mu}=7.215, \varepsilon_{2 \mu}=-0.548$. Note that the energy of the second final particle is negative and the energy of the first final particle contrary to the limit obtained in 11] is larger than the energy of initial particles as it must be in the case of a Penrose process 7,8 . What is the reason of this contradiction? Let us investigate the problem carefully.

Note that if one neglects the states with negative energy in ergosphere energy extracted in the considered process cannot be larger than the initial energy of the pair of particles at infinity, i.e. $2 m$. The same limit $2 m$ for the extracted energy for any (including Penrose process) scattering process in the vicinity of the black hole was obtained in [11]. Let us show why this conclusion is incorrect.

If the angular momentum of the falling particles is the same then (see (24)) one has the situation similar to the usual decay of the particle with mass $2 m$ in two particles with mass $\mu$. Due to the Penrose process in ergosphere it is possible that the particle falling inside the black hole has the negative relative to infinity energy and then the extracted particle can have energy larger than $2 m$.

The main assumption made in [11] is the supposition of the collinearity of vectors of 4-momenta of the particles falling inside and outside of the black hole in limiting case $A=1, l_{1}=2$ (see (9)-(11) in [11]). The authors of [11] say that these vectors are "asymptotically tangent to the horizon generator".

In the limiting case the expressions $d t / d \tau, d \varphi / d \tau$ of the components of the 4 -velocity of the infalling particle (6), (7) go to infinity when $r \rightarrow r_{H}$, but $d r / d \tau$ goes to zero. In spite of smallness of $r$-components in the expression of the square of the 4-momentum vector they have the factor $g_{r r}$ going to infinity at the horizon. For example from (1), (8) it is easy to see that

$$
g_{r r}(x) u_{(1)}^{r} u_{(1)}^{r} \rightarrow-2, \quad x \rightarrow x_{H} .
$$

So putting them to zero can lead to a mistake. To see if $u_{(1)}$ and $v_{(1)}$ are collinear it is necessary to put the coordinate $r$ of the collision point to the limit $r_{H}=M$ and resolve the uncertainties $\infty / \infty$ and $0 / 0$. For the falling particle $\varepsilon=1, l=2$ the expressions for components of the 4 -vector $u$ can be easily found from (6) $-(8)$. For the particle outgoing from the black hole due to exact solution on the horizon (25) one puts $\varepsilon_{1 \mu}=l_{1 \mu} / 2+\alpha$, where $\alpha$ is some function of $r$ and $l_{1 \mu}$, such that $\alpha \rightarrow 0$ when $r \rightarrow r_{H}$. Putting this $\varepsilon_{1 \mu}$ into (6)-(8) one gets for $x=r / M \rightarrow 1$

$$
\begin{gathered}
\frac{v_{(1)}^{t}}{u_{(1)}^{t}}=\frac{v_{(1)}^{\varphi}}{u_{(1)}^{\varphi}}=\frac{\alpha}{x-1}+\frac{l_{1 \mu}}{2}, \\
\frac{v_{(1)}^{r}}{u_{(1)}^{r}}=-\sqrt{\frac{2 \alpha^{2}}{(x-1)^{2}}+\frac{2 l_{1 \mu} \alpha}{x-1}+\frac{3}{8} l_{1 \mu}^{2}-\frac{1}{2}} .
\end{gathered}
$$


Due to the condition $d t / d \tau>0$ (movement forward in time) the necessary condition for collinearity is that both (27) and (28) must be zero, which is not true. The notion of the asymptotic behaviour of 4-vectors on the horizon (asymptotic collinearity) needs delicate mathematical analysis. One must find the limit of the norm of vectors. But this norm is defined by the scalar product of vector on itself and it contains finite terms of the type (26) neglected by the authors [11, when they put the hypothesis that the momentum of ejecta particle is $\lambda k$. This hypothesis means that the $r$-components in (28) are taken as identically zero and (27) is enough for collinearity, however (26) and (28) show that this is incorrect. This leads to the conclusion that the considerations of the authors of [1] for scattering exactly on the horizon can not be used for the real situation of particle scattering close to the horizon.

1. A. A. Grib and Yu. V. Pavlov, Mod. Phys. Lett. A 23, 1151 (2008).

2. The Pierre Auger Collaboration, Science 318, 938 (2007)

3. A. A. Grib and Yu.V. Pavlov. Grav. Cosmol. 15, 44 (2009)

4. R. Penrose, Rivista Nuovo Cimento I, Num. Spec., 252 (1969)

5. K. Greisen, Phys. Rev. Lett. 16, 748 (1966)

6. G. T. Zatsepin and V.A. Kuzmin, JETP Lett. 4, 78 (1966).

7. T. Piran, J. Shaham, and J. Katz, Astrophys. J. Lett. 196, L107 (1975)

8. T. Piran and J. Shaham, Phys. Rev. D 16, 1615 (1977).

9. M. Banados, J. Silk, and S. M. West, Phys. Rev. Lett. 103, 111102 (2009)

10. E. Berti, V. Cardoso, L. Gualtieri, F. Pretorius, and U. Sperhake, Phys. Rev. Lett. 103, 239001 (2009).

11. T. Jacobson and T.P. Sotiriou, Phys. Rev. Lett. 104, 021101 (2010)

12. S. Chandrasekhar, The Mathematical Theory of Black Holes, Oxford Univ. Press, Oxford, 1983.

13. K. S. Thorne, Astrophys. J. 191, 507 (1974). 